\title{
The Barriers to and Benefits of Use of ICT for People with Visual Impairment
}

\author{
Kristin Skeide Fuglerud \\ Norsk Regnesentral, Gaustadalléen 23, P.O. Box 114, Blindern, NO-0314 Oslo, Norway \\ kristin.skeide. fuglerudenr.no
}

\begin{abstract}
This paper reports results from a focus group interview and a field study, which includes 28 visually impaired PC users in Norway. The main goal of the study was to identify benefits of, and barriers to, use of ICT for the visually impaired, and to propose measures to remove barriers. The use of Internet services, mobile phones, kiosks, ticket machines, ATMs, and queuing management systems, were studied. Visually impaired users' encounters with technology were investigated through a focus group interview, observation of task-solving activities, and semi-structured interviews. The analysis revealed that several commonly used ICT services, such as online banking, electronic forms, and learning material have major accessibility problems. The first barrier is often mechanisms for registration and authentication. The proliferation of inaccessible everyday technologies, unstable systems, and lack of training constitutes other major challenges. Based on the findings some suggestions for further development and research priorities are suggested.
\end{abstract}

Keywords: accessibility, universal design, visually impaired, assistive technology, self-service terminals, queuing systems, user diversity.

\section{Introduction}

The rapid development and widespread adoption of Information and Communication Technology (ICT) has fundamentally changed almost every aspect of our lives. The development affects how we perform various tasks in our daily lives. Industry leaders and policy makers have acknowledged that technology has the capacity to increase the quality of people's lives by improving the effectiveness of teaching and learning, and the productivity of industry and governments. Moreover, because much of an individual's future success may rely on their ability to use ICT, access to ICT and opportunities to learn how to use ICT must be made as equitable as possible. The question is how to achieve this for the most excluded groups.

A national survey about e-democracy found that Visually Impaired (VI) people in Norway used ICT significantly less often than the rest of the population [1]. People with various disabilities (vision, hearing, movement, and dyslexia) participated in this survey. Moreover, VI respondents stated that ICT is difficult to use, more often than did respondents with other impairments. It was concluded that Internet technology seems to be exclusionary to the VI, and that it is a challenge to include the VI through digital channels. 
Another survey was commissioned by the Norwegian Association for the Blind and Partially Sighted (NABP), and was conducted by a market research company [2]. This survey dealt with VI people's encounters with everyday technology such as ATMs, queuing systems, ticket machines, and elevators. It revealed that everyday technology offered many challenges for the VI. For example, it showed that half of the 400 respondents felt great discomfort in situations encountering queuing systems. It also showed that a majority of the VI respondents with an ATM card had given out their pin codes to other people when using ATMs, because they could not operate the machines on their own [2].

As a consequence of these alarming findings, we applied for funds to investigate the situation more in-depth in collaboration with the NABP. It is necessary to have detailed knowledge about what challenges, problems, and benefits the VI have when using technology in order to apply effective measures to include the VI in the information society. In this paper we present some important findings from this exploratory study to investigate the role of ICTs in the lives of people who are blind or partially sighted, i.e., people with visual impairments. A full report can be found in Norwegian [3].

\section{Related Work}

Providing the VI with Assistive Technology (AT), such as screen-reading and magnifier technologies, is necessary, but insufficient to make VI individuals able to use ICTs. It is well documented that Internet services are often designed in a way that makes it difficult to use for persons with visual impairments [3-6]. Similarly, educational systems and learning management systems are found to have poor accessibility for the VI [7, 8]. Furthermore, during the last few years, researchers have pointed to the need for more accessible web 2.0 systems $[6,9]$. The research so far has also shown that web site compliance with standards and guidelines is a necessity, but does not necessarily provide Internet services that are usable for visually impaired people $[10,11]$. Thus, existing accessibility guidelines are criticized for focusing solely on technical accessibility to ensure that web pages are compliant with assistive devices [12]. New guidelines focussing on a combination of usability and technical accessibility are emerging $[10,13]$.

One in-depth case study describes how a blind person interacted with a variety of artefacts in her own home [14]. The aim of the study was to understand how a blind person mentally represents the environment, and how she applied alternative options in order to accomplish tasks. There are also some recent studies related to how to present public transport information in an accessible way on smartphones [15] [16].

To summarize, an increasing number of studies document VI people's challenges when using Internet services. It seems, however, to be very scant literature with regard to VI people's encounters with everyday technologies such as mobile devices, kiosks, ticket machines, ATMs, and queuing management systems. Since technology is continuing to penetrate our daily lives, more knowledge about the accessibility of these technologies is needed. 


\section{Method}

Since the aim of the study was to learn more about the use of technology among VI people, and in particular to learn more about their challenges and barriers, we utilized a broad and exploratory approach in which several qualitative methods were combined; namely a focus group interview and a field study with 28 visually impaired PC users. This approach would give the possibility to go in-depth on the experiences and individual challenges of the participants' everyday life, and also the possibility to bring to light issues and topics that were not known in advance. Below, the methods and procedures used are described in more detail.

Focus group interview: An initial focus group meeting with 10 participants was conducted to provide input about the research theme, and to form the basis for the field study. People with different experience and knowledge about how VI people use technology participated. There were researchers, suppliers of and teachers in Assistive Technology (AT), visually impaired people themselves, and employees at the Norwegian Association for the Blind and Partially Sighted. A thematic interview guide was sent out beforehand and followed during the meeting. The focus group meeting was audio recorded and transcribed.

Field study: The field study was designed based on a literature study and input from the focus group interview. The aim was to give a broad, but realistic picture of use of ICT among VI people. Since people can get very accustomed to their obstacles, adapting to them and working around them to such a point that they become practically unaware of them, we wanted to combine interviews with observations. We did not have time and resources to observe users while using all sorts of everyday technology such as kiosks, ticket machines, ATMs, and queuing systems. Therefore, we chose to have the participants solving some common PC tasks. From previous studies, we have experienced that people often forget details about their own ICT behaviors. We, therefore, hoped that the PC interaction session would help to spur memories of interaction with other technologies as well. An interview guide and a set of PC tasks were developed. Access to, and familiarity with, a PC with necessary Assistive Technology was a precondition for participation in the field study.

Participants: Visually Impaired people are a heterogeneous group. They use a variety of AT depending on the degree of their impairment. People who are blind typically use screen readers, as do some people with very low vision. A screen reader converts the text on a computer screen into Braille or into spoken words using a synthesized voice. People who are partially sighted often use screen magnifier software. With screen magnifier software the user can take a small area of the computer screen and make it larger. Screen magnifiers also let users invert or adjust colors and contrast in order to support their type of sight loss in an optimal way. Since we wanted to observe users while using their AT, we chose to visit them where they normally keep and use their equipment, either at home, at an office, or at school.

The participants were recruited through the Norwegian Association for the Blind and Partially Sighted (NABP), and their knowledge about their members was essential in order to get a distribution with respect to degree of sight loss, the type of AT they used, computer experience, education, and occupational situation. A detailed 
information letter about the study test procedure, expected duration, and the need for a PC with appropriate AT was sent to the members. The participants were given a monetary compensation of NOK 500 (which is a normal level of compensation for participation in usability studies in Norway). From the volunteers, 30 people were selected for the study of which 28 actually participated; these included twelve males and sixteen females from the eastern part of Norway. Fourteen participants were blind, and fourteen characterized themselves as partially sighted. Seven participants were students, 11 participants had a job, and 10 participants did not work nor study. The age range of the participants was quite smoothly distributed between 17 and 60 years old. The participants covered the whole spectrum of ICT skills and experience, from novice to highly experienced expert user. Seventeen participants had higher education at a college or university level.

Materials and tasks: A semi-structured interview guide and tasks were developed based on a literature review, background information, such as statistics on ICT, and input from a focus group. The main criteria for selection of tasks were that it was a common task that many people do, that it was aimed at everybody and did not require any special knowledge or training. The tasks included the use of timetables and schedules for public transport, buying a train ticket, finding public information, finding learning material using their university or educational institution's web pages, finding the schedule of a radio program, using a public portal to find information about cultural events in their county, using a standard form for job applications, and reading news in a local newspaper. The use of an Internet bank was considered an important task example, but was not included, because of privacy concerns. It was, however, included in the interview guide. The intention was that the interviews would give a picture of how ICT is experienced in the daily life of the participants, while observation of task-solving activities would provide concrete examples of how VI people experience and work with common ICT-related tasks, as well as spur their memory and association of interactions with other technologies.

Procedure: Research conventions on ethics and privacy of the participants were followed. The whole interview and observation session was audio recorded. The session started with the collection of some demographic and background information such as age, gender, occupation, ICT experience, details about the participant's impairment, and the type of Assistive Technology available to them. The interview and observation was conducted in a relaxed tone, where the participant could go into detail on issues they found important and relevant along the way. In the observation section, participants would select tasks they could relate to from the sample of test tasks that were provided. Dependent on their skills and speed, they would do one or more tasks within the upper time limit. Each interview and observation lasted from $1 \frac{1}{2}$ to 2 hours. While completing the tasks, the candidates were encouraged to think aloud. The researchers noted problems, concerns, and also the participant's actions and comments. In case the participant was unable to continue on their own, they would get hints from the researcher.

Data collection and analysis: A fairly detailed set of minutes from each interview and observation session was written based on notes and audio recordings. Parts of the sessions were transcribed; and, a thematic analysis was performed. Minutes from all the sessions were re-read with the aim of forming themes, and then sections from the 
individual minutes were sorted into categories according to the different themes. During the analysis new themes and categories emerged. The results described in the next section are based on this thematic analysis.

\section{Results}

Web pages, learning material, and electronic forms: From the interview sessions, the participants seemed quite positive towards web accessibility. However, during the observation of the task-solving activity we found that most participants had severe problems in completing their tasks. For example, booking railway tickets online was, in practice, totally inaccessible to the VI. The general conclusion was that even though VI users are able to use some web pages, most web pages had poor accessibility. Even web pages with a high score on a yearly web page quality evaluation performed by the Norwegian Agency for Public Management and Government, had poor accessibility. Often there were inaccessible elements on the pages such as inaccessible documents and electronic forms with inaccessible formatting.

Online banks, registration, and authentication: Because of security requirements, online banks often have authentication solutions that are more complicated than authentication solutions found in other Internet services. The most common authentication method in Norway, BankID, applied a java applet which was inaccessible to screen reader users. This solution also required that the user enter a code from a separate code-generation token in combination with a password or PIN. Although accessible code generation tokens exist, only one participant had been offered this. Furthermore, even if the participants got help to authenticate they would be afraid of making mistakes, because of poor accessibility within the Internet bank. Also, sometimes it is necessary to read information on paper bills such as account numbers and verification numbers. E-billing will increasingly address these problems. However, as a result of all the current obstacles, only a couple of the participants used online banking themselves. Typically, a family member would do the banking task for them. In general, registration and authentication were commented on as being a major barrier for the VI. Many services require a security check, such as the widespread use of CAPTCHA codes, however, some websites now offer audio CAPTCHA, but this solution seems to be quite difficult to use as well.

Self-service terminals and ATMs: Many services are transferred from humans to selfservice terminals such as kiosks, ticket machines, and ATMs. It is quite clear that these technologies are often difficult or impossible to use by the VI. A common strategy among the participants was to learn the tapping sequence of buttons on specific terminals by heart, which they could not avoid using during everyday life. However, this strategy depended on the terminal having distinct and tactile buttons, and a stable interface. We got some vivid descriptions of how participants found it extremely stressful if the queue behind them grew as they kept struggling with a selfservice terminal. Thus, they tried to avoid such terminals, for example, by purchasing tickets in advance at a counter or with the aid of a sighed person. Eight participants felt that it had become significantly harder to get manual service at a counter or by 
telephone. Several expressed concern about the general trend towards more selfservice terminals, and in particular towards the increased use of touch panels, which in general excludes access for the VI.

ATMs were also regarded as inaccessible, although thirteen participants had used them However, nearly all participants found it okay to use card terminals in stores. It was commented that the number keypads on card terminals had become more standardized, e.g., with regard to the placement and order of the various buttons (such as numbers, OK, and Cancel). Therefore, it was most convenient to withdraw money while shopping. Six participants mentioned that they knew one or a few ATMs in their neighborhood that they could use, because they had learned the dialogue on these ATMs by heart; they knew exactly what buttons they needed to press, and in what sequence. Some months before the fieldwork, one Norwegian bank had introduced a speech interface with their ATMs. To use this terminal, the VI user could bring their own earplugs, plug them into the terminal, and listen to the user interface dialog. At the time of our fieldwork only two participants had tried this solution, and both were highly satisfied with it. One of them was excited because he had been able to withdraw foreign currency before a holiday on his own, without help. He pointed out that he would never learn this function by heart, as it was seldom used. Therefore, being able to use it without previous training was a huge improvement.

Queuing management systems: It was a little surprising for us to discover that the increased use of queuing management systems in various offices and shops created so much frustration and discomfort for our participants. Ten participants found it very uncomfortable to enter premises with queuing systems, and several told us about unpleasant episodes regarding these systems. They used strong expressions like "I hate it", and "it is terrible" or "embarrassing". Very few participants would walk past the queue to the counter even though the queuing system was impossible for them to use. The majority of the participants said they would just stand there and hope the staff would discover them. Being in a familiar place, or having a visible symbol of the visual impairment, such as a guiding dog or a cane, could make it easier to be discovered, and to get help from the staff. The overall consequence of the increased use of queuing management systems was that participants had become more dependent on bringing an assistant or getting assistance when doing their errands in such places.

Mobile devices: All our participants used a cell phone. Although having a cell phone is very common among the VI, this technology also has its challenges. Since 2006, VI people in Norway can apply for financial support from the public welfare system to get AT, which reads out menus and text messages using synthetic speech synthesis. There are also solutions that make it possible to transmit the text to a handheld Braille display. This technology has made a positive impact, which is huge in the lives of many of the participants. In particular the ability to read, write, and send a SMS, made participants feel less dependent on others. It was noted, however, that there was a relatively small range of mobile phones that were usable with text-to-speech AT, and that it was difficult to find information about which model to choose. It was pointed out that it is difficult to test the font sizes and menus of a mobile phone in a shop, especially since it would be demonstrated without the AT. On the positive side, 
participants expressed optimism with regard to the vast possibilities of new small devices such as GPS, notebooks, and smartphones with AT.

Vulnerability towards technical problems, changes, and updates: Visually impaired people share many technological challenges with people who are sighted, but there are nevertheless some conditions that are specific to the VI. During the task-solving part of the study we observed remarkably many occurrences of unexplainable slow performance, hanging computers, and even computer crashes. The interviews confirmed that frequent technical problems were the rule, rather than the exception. The most obvious explanation is that VI users typically use a wider range of hardware and software than sighted ICT users. This leads to several complicating circumstances for the VI. First, the technical problems often go beyond one single tool or equipment, and the more components there are, the more potential causes to the problem. Second, often the AT itself is affected by the technical problems, and then there are no means for the VI to read the error messages and instructions that would help them solve the problem. Third, all computer users have to face updates and new versions from time to time. However, because AT adds an extra layer, the VI have to face more frequent updates, and every update involves more elements, having to check out that the new configuration works and to learn new features with the AT. As a consequence, a VI computer user does not only need more competence in order to use ICT at the same level as a sighted person, it is also important that he or she has adequate equipment in terms of processing power, memory, robustness, and interoperability.

One third of the participants expressed that they were totally dependent on family members or friends with technical knowledge or ICT education to help them with technical difficulties. It appears to be essential that there are people around the VI, such as teachers, family, or friends, who have the technical skills required to get things to work. All these factors contribute to a demanding situation for the VI in terms of learning and problem-solving capacity. Therefore, it is no wonder that many of the VI participants in this study expressed a need for more training and support.

Need for training: Because of the extra layer that AT constitutes, there are extra cognitive and learning demands on the VI when using ICT. Therefore, there is a need for training on several levels. First, visually impaired people have less opportunity than others to read manuals and error messages, since these manuals and messages are often inaccessible. Therefore, the VI must be trained to handle issues covered by such inaccessible material. Secondly, there is a need for training for how to do customizations and optimize settings in the assistive hardware and software. Third, there is a need for training for the use of AT in combination with other software, and last but not least, the frequency of change in the technology configuration may be higher for a VI person, and thus demanding more frequent training. Some participants mentioned that they missed having a place to find and share practical experiences and information related to the use of ICT. They envisioned a place for where VI could share vendor independent reports on usability and accessibility of products and services, tips and tricks on how to solve problems, and descriptions or reports on new and useful technologies. 


\section{Discussion}

Although the sample of respondents is not random in the statistical sense, the study gives a broad picture of real-world experiences. The fact that over half of the participants had higher education only emphasizes the challenges. The study has revealed a complex and sometimes contradictory picture. On the one hand, the development of ICT has made a tremendous difference in the lives of VI individuals by improving education and employment opportunities, enhancing social interaction, and facilitating independence. Also, the participants in this study often showed an impressive learning ability, memorizing capability, and ability to find new and original ways of using technologies. The use of e-mail and SMSs together with AT and talking ATMs are examples of technological advances that have significantly increased the opportunity for the VI to participate in an information society. On the other hand, there are still a number of ICT-related obstacles that VI people face in interacting with web pages, educational material, technology at work, and everyday technology. Moreover, one of the main challenges for the VI is the dynamic and changing nature of many interactive systems. As described, changes and updates require much effort from VI users and their supporters in terms of updating, interoperability, learning and problem-solving capacity.

All these factors contribute to a situation where the VI is frequently totally dependent on the help from sighted persons. The obstacles are so extensive that VI people are far from having equal opportunities in the information society, as do their sighted peers. Based on the findings in this study some areas for further development and research emerge. These areas are described in the following.

Accessibility guidelines with corresponding development tools: The work on guidelines with corresponding tools for making web technology both technically accessible and usable should continue. The international standard ISO 9241-171:2008 has incorporated usability into their accessibility concept. This standard requires that in order for an interactive system to be accessible, disabled users must be able to use it to achieve a specific goal [14]. New guidelines, which are more in line with the ISO definition of accessibility, i.e., focusing on a combination of usability and technical accessibility, are emerging. However, the growing quantity of recommendations contained within each set of guidelines, coupled with the general lack of (semi-) automated tools to support the developers in adhering to the guidelines, may contribute to a slow adoption of these guidelines.

Accessible identification and security systems: Accessible and usable registration and authentication is a prerequisite for access to many Internet services and interactive systems. User identification is also a prerequisite for individual profiling, and thus, for the possibility of providing services that can adapt to the needs of the individual user. Ordinary accessibility guidelines may not be applicable when designing security solutions [17]. Therefore, dedicated work to make security solutions both accessible and usable is needed.

Accessible interactive learning material: The participants in this study called for more training. This need comes as a natural consequence of the complexity of their technological environment, including AT and the fact that much of the existing learning materials, such as guides, videos, screencasts, etc., are inaccessible. Thus, 
there is a need for accessible interactive learning materials on the various technologies that VI users need to learn and use.

Accessible social networks and web 2.0 technology: In addition to the obvious social function that social networks have, which is a good enough reason in itself to require accessibility, such tools can play an important role as a means to share knowledge and competence among people. Participants in our study called for a way to find and share information and experiences about technology use. Web 2.0 technologies has the potential as a tool for empowerment by providing accessible places where the VI together with their teachers, providers of AT, and others, can share relevant information, material-related to accessibility, and use of technology.

Accessible mobile web: An interesting side effect of the increasing number of people using handheld devices and smartphones for web browsing is the emergence of mobile web interfaces. It seems that mobile web interfaces often are more accessible for visually impaired people than traditional web interfaces. For example, after the emergence of the mobile web interface to Facebook (i.e., the m.facebook.com), many VI people found that they were able to use Facebook through this interface, because it is more accessible than the original interface [18]. Therefore, it seems appropriate to further develop and emphasize the accessibility aspects of mobile web interfaces.

Accessibility in the internet of things. Because of the possibility for VI users to use their mobile or handheld device with AT, one can envision that the mobile or handheld device can function as a "front-end" to everyday technologies. For example, information from a ticket machine, or from a web page that is associated with the ticket machine, could be transferred and presented on their mobile device in an accessible way. The advantage is that the VI user would be familiar with the ways to interact with his or her own device and its features, including any necessary AT. Research into accessible interactions with everyday technology through the Internet of Things is an area with huge potential benefits to various user groups.

\section{Conclusion}

It is increasingly recognized that being able to use ICT can make a tremendous difference in the lives of individuals with visual impairments, improving educational and employment opportunities, enhancing social life, and facilitating independence. This study has, however, showed that the VI experience major challenges in using ICTs in their daily life. Many Internet services, such as public websites, online banking, and electronic forms, had poor accessibility. Inaccessible mechanisms for registration and authentication were a major barrier to the use of various Internet services. The proliferation of self-service terminals and queuing management systems constituted another major barrier for the VI. Unstable systems and lack of training are among other identified challenges.

Based on these findings six areas for further research and development were identified: 1) Continuing the development of better guidelines for accessible and usable Internet services with corresponding development tools; 2) Accessible registration and authentication mechanisms; 3) Accessible and interactive learning materials; 4) Accessible social networking services and web 2.0 tools; 5) Accessible mobile web devices; and 6) Accessibility in the Internet of Things. 
Acknowledgments. The work with this paper has been partly financed by the Norwegian research council through the e-Me project, and NordForsk through the IVIS project. The study work was conducted within the project "The ICT barriers for the visually disabled" [19]. Special thanks to all the participants in the study, and to my colleague Ivar Solheim for collaboration in the study and for constructive feedback on this paper.

\section{References}

1. Hanssen, G.S., Winsvold, M.: Local e-democracy: On ICT mediated participation among people with disabilities (in Norwegian) NIBR (2006)

2. Rønning, T.: Everyday technology for all - or nearly all (in Norwegian). Norges Blinde, vol. 2004. NABP, Oslo (2004)

3. Fuglerud, K.S., Solheim, I.: ICT-barriers for the visually impaired (in Norwegian). Report, Norwegian Computing Center (2008)

4. Lazar, J., Allen, A., Kleinman, J., Malarkey, C.: What Frustrates Screen Reader Users on the Web: A Study of 100 Blind Users. International Journal of Human-Computer Interaction 22, 247-269 (2007)

5. Zúnica, R.R., Clemente, V.Á.: Research on Internet use by Spanish-speaking users with blindness and partial sight. Universal Access in the Information Society 6, 103-110 (2006)

6. Hailpern, J., Guarino-Reid, L., Boardman, R., Annam, S.: Web 2.0: blind to an accessible new world. In: Proceedings of the 18th International Conference on World Wide Web, pp. 821-830. ACM, Madrid (2009)

7. Dini, S., Ferlino, L., Gettani, A., Martinoli, C., Ott, M.: Educational software and low vision students: evaluating accessibility factors. Universal Access in the Information Society 6, 15-29 (2007)

8. Buzzi, M., Buzzi, M., Leporini, B.: Accessing e-Learning Systems via Screen Reader: An Example. In: Jacko, J. (ed.) Human-Computer Interaction. Interacting in Various Application Domains, vol. 5613, pp. 21-30. Springer, Heidelberg (2009)

9. Buzzi, M.C., Buzzi, M., Leporini, B., Mori, G., Penichet, V.M.R.: Accessing Google Docs via Screen Reader. In: Miesenberger, K., Klaus, J., Zagler, W., Karshmer, A. (eds.) ICCHP 2010. LNCS, vol. 6179, pp. 92-99. Springer, Heidelberg (2010)

10. Leporini, B., Paternò, F.: Increasing usability when interacting through screen readers. Universal Access in the Information Society 3, 57-70 (2004)

11. Rømen, D., Svanæs, D.: Validating WCAG 1.0 and WCAG 2.0 through Usability Testing with Disabled Users. In: Sandnes, F.E., et al. (eds.) Unitech 2010, pp. 168-189. Tapir Academic Publishers, Oslo (2010)

12. Murphy, E., Kuber, R., McAllister, G., Strain, P., Yu, W.: An empirical investigation into the difficulties experienced by visually impaired Internet users. Universal Access in the Information Society 7, 79-91 (2008)

13. Arnim, D., Piuzzi, B., Nam, C., Chung, D.: Guidelines for the development and improvement of universal access systems for blind students. In: Stephanidis, C. (ed.) HCI 2007. LNCS, vol. 4554, pp. 603-612. Springer, Heidelberg (2007)

14. Shinohara, K., Tenenberg, J.: A blind person's interactions with technology. Commun. ACM 52, 58-66 (2009)

15. Minifie, D., Coady, Y.: Getting mobile with mobile devices: using the web to improve transit accessibility. In: W4A 2009, pp. 123-126. ACM, Madrid (2009) 
16. Azenkot, S., Fortuna, E.: Improving public transit usability for blind and deaf-blind people by connecting a braille display to a smartphone. In: ASSETS 2010, pp. 317-318. ACM, Orlando (2010)

17. Fritsch, L., Fuglerud, K.S., Solheim, I.: Towards Inclusive Identity Management. Identity in the Information Society 3, 515-538 (2010)

18. Tollefsen, T., Dale, Ø., Berg, M., Nordby, R.: Connected! A paper about the disabled and the use of social media., MediaLT (2011)

19. Norwegian Computing Center: ICT barriers for the Visually Disabled, http://www.nr.no/pages/dart/project_flyer_synshemmedes_ikt_b arrierer 